Check for updates

Cite this: RSC Adv., 2018, 8, 39992

\title{
Scalable and facile synthesis of stretchable thermoelectric fabric for wearable self-powered temperature sensors $\uparrow$
}

\author{
Minhyun Jung, ${ }^{a}$ Sanghun Jeon (D) *a and Jihyun Bae (D) *b
}

\begin{abstract}
Wearable sensor systems with ultra-thinness, light weight, high flexibility, and stretchability that are conformally in contact with the skin have advanced tremendously in many respects, but they still face challenges in terms of scalability, processibility, and manufacturability. Here, we report a highly stretchable and wearable textile-based self-powered temperature sensor fabricated using commercial thermoelectric inks. Through various combinations of poly(3,4-ethylene dioxythiophene)-poly(styrene sulfonate) (PEDOT:PSS), silver nanoparticles (AgNPs), and graphene inks, we obtained linear temperature-sensing capability. The optimized sensor generates a thermoelectric voltage output of $1.1 \mathrm{mV}$ for a temperature difference of $100 \mathrm{~K}$ through a combination of PEDOT:PSS and AgNPs inks and it shows high durability up to 800 cycles of $20 \%$ strain. In addition, the knitted textile substrate exhibits temperature-sensing properties that are dependent upon the stretching directions. We believe that stretchable thermoelectric fabric has broader potential for application in human-machine interfaces,

health-monitoring technologies, and humanoid robotics.
\end{abstract}

Received 8th August 2018

Accepted 27th November 2018

DOI: $10.1039 / \mathrm{c} 8 \mathrm{ra0} 6664 \mathrm{~g}$

rsc.li/rsc-advances

\section{Introduction}

Stretchable electronics and sensors have been the focus of tremendous interest due to their potential for use in a variety of applications, such as direct human-machine interfaces, healthmonitoring devices, motion-tracking sensors, artificial electronic skin, and energy-related devices..$^{1-6}$ Energy harvesting systems have emerged as attractive and potentially sustainable power sources. They will also allow sensor systems to be independently and sustainably self-powered using piezoelectric, triboelectric, pyroelectric, and thermoelectric effects. ${ }^{7-11}$

In particular, wearable device systems have to fulfil several requirements including those of sensitivity and accuracy, softness, biocompatibility, high cycle life, light weight, multipoint sensor arrays or networks, and being conformal to the surface of skin. Recently, there has been much progress in flexible and stretchable electronic devices using organic semiconductors, ultra-thin silicon membranes, metal-based films, serpentine lines or liquid metals, carbon nanotubes, silver nanowires, and intrinsically conductive polymers. ${ }^{12-16}$ While these materials and structures provide excellent stretchability, devices

${ }^{a}$ School of Electrical Engineering, Korea Advanced Institute of Science and Technology (KAIST), 291 Yuseong-gu, Daejeon, 34141 Korea. E-mail: jeonsh@kaist.ac.kr

${ }^{b}$ Department of Clothing \& Textiles, Hanyang University, 222 Wangsimni-ro, Seongdong-gu, Seoul 04763, Korea. E-mail: jbae2@hanyang.ac.kr

$\dagger$ Electronic supplementary information (ESI) available: SEM images of the knitted fabric substrate printed with AgNPs after stretching. See DOI: $10.1039 / \mathrm{c} 8 \mathrm{ra06664g}$ fabricated from these materials are typically realized by lithographic or spin or spray coating techniques; the structurefabrication processes are complex and either expensive or incompatible with large-scale fabrication. ${ }^{17,18}$

On the other hand, as the ultimate wearable platform, textile-based electronics have the proven advantages of their intrinsic properties, such as light weight, accessibility, comfort, breathability, and stretchability., ${ }^{3,19-21}$ To date, various wearable strain- and pressure-sensing devices have been demonstrated on textile platforms. These devices have been demonstrated to achieve highly sensitive strain/touch/pressure sensors performance, and wireless user interfaces have been realized using entirely polymeric conducting fibers. ${ }^{3,22-25}$ Recently, based on highly stretchable fiber-based electronic textiles, a strain/ pressure sensor was reported for full-range human motion detection, health monitoring, and 2D force mapping by Chen et al. ${ }^{26}$ Advanced textile-manufacturing technologies can provide a variety of fabrication methods to produce sensory systems that are low-cost, mechanically reliable, flexible, and suitable for industrial mass production. Nevertheless, due to their fabric construction technology, woven fabrics composed of horizontally and vertically interlacing threads, show limited stretchability. On the contrary, knitted fabrics are composed of consecutive rows of interconnected loops that provide the potential to be easily deformed. Further, the space existing in each loop enables the knitted fabric to be stretched in all directions..$^{27,28}$

Previously, electrical conductivity in textiles has been achieved through several methods, such as metallization, 
electroless deposition, electrodeposition, and chemical coating, depending on the desired level of conductivity and durability as well as the process requirements. ${ }^{29}$ In recent years, printing has been widely explored in the textile and flexible electronics industries because it offers a low-cost, simple process that is suitable for large-scale production and can be conducted at low temperatures. ${ }^{30,31}$ In this regard, printed electronics on typical substrates, such as papers or textiles, can be used in a variety of industrial applications, including batteries, transistors, displays, photovoltaics, and sensors.

In this study, we demonstrated a new concept of making stretchable self-powered temperature sensor arrays by using a well-established printing technique on knitted fabric with commercialized thermoelectric materials. We employed the thermoelectric effect that utilizes temperature gradients across a printable material to drive the electric current, usually called the Seebeck effect. It worked as a self-powered temperature sensor that automatically detects the temperature difference across the device without batteries or other power sources. To obtain good temperature sensing performance over a wide range, we prepared three combinations of commercial printable inks of a conductive polymer PEDOT:PSS, silver nanoparticles (AgNPs), and graphene and formed a closed-loop thermocouple system composed of a printed pattern. By taking advantage of the knitting technology, the self-powered temperature sensor possesses high elasticity, flexibility, and stretchability in all direction. In addition, in a cyclic test up to $20 \%$ strain, the temperature sensor exhibited excellent durability with only a $7 \%$ difference in voltage. Since the core materials used in this study including a commercial knitted fabric and conductive inks are inherently flexible, cost-effective, and suitable for largescale industrial manufacturing, our work can make a significant contribution to the development of flexible and stretchable sensor applications.

\section{Experimental section}

\section{Materials}

A commercially available knitted textile with a jersey structure composed of $80 \%$ 150-denier polyester and 70-denier cotton was prepared as the stretchable substrate (Fig. 1b). We used various conducting inks composed of PEDOT:PSS, AgNPs and graphene (Sigma-Aldrich) as the core thermoelectric materials in the temperature sensor. To enhance the conductivity, the PEDOT:PSS solution was mixed with dimethyl sulfoxide (DMSO) and FC-5120 (3M fluorosurfactant) (94: $5: 1$ (w/w/w)). DMSO and fluorosurfactant have been reported to enhance the conductivity of PEDOT:PSS. Also, the use of a fluorosurfactant reportedly improves the wetting properties of PEDOT:PSS solution, which enables the facile deposition of a highly conductive PEDOT:PSS layer on a variety of stretchable substrates. ${ }^{32,33}$ The PEDOT:PSS was stirred at high speed $(>500$ $\mathrm{rpm}$ ) while various amounts of DMSO were added and blended with the FC-5120. All of the polymer mixtures were filtered through a syringe filter $(0.5 \mu \mathrm{m}$ pore size $)$ after mixing. Three combinations of prepared inks, namely, PEDOT:PSS and AgNPs, PEDOT:PSS and graphene, and AgNPs and graphene, were placed on simple patterned polyimide masks and were spread across the openings by a squeegee, respectively. Finally, the stencil was lifted off the substrate leaving the designed pattern with overlapping areas of $4 \mathrm{~mm}^{2}$ deposited on the substrate. ${ }^{19}$ Once each ink had been printed on the substrate, the samples were cured on a hot plate at $120{ }^{\circ} \mathrm{C}$ for $30 \mathrm{~min}$. The curing conditions were optimized as described by previous researchers..$^{30,31}$

\section{Device characterization}

The electrical characteristics of the thermoelectric sensor were assessed using a universal measurement probe system (Tera Leader UMP-1000, Korea). For two different temperature set-up of the sensor, the junction area was placed on the hot-plate to apply a range of the temperature and the thermocouple leg parts were kept at room temperature. The temperature difference could be defined by measuring both the hot-plate and room temperatures. To evaluate the device performance of the thermoelectric sensor, a high-resolution digital multimeter (Keysight 34401A) was employed to detect its output Seebeck voltage. The real-time mapping data of the 5 by 5 sensor array system with the unit area of $25 \mathrm{~mm}^{2}$ was characterized by using a LabVIEW-based evaluation system (USB-6218).

\section{Results and discussion}

Fig. 1a shows a schematic illustration of the fabrication process of the textile-based temperature sensor using stencil-printing technology. The device pattern printed on the textile substrate is composed of a junction area overlapped with $\mathrm{p}$ - and n-type conductive inks and two legs that connected electrically to determine the thermoelectric properties. The highly stretchable feature of the knitted fabric in multiple directions, including the vertical, horizontal, and diagonal directions, is shown in Fig. 1b. As shown in Fig. 1c (left), a knitted fabric consists of several consecutive rows of inter-looping a head and two legs. The knitted loops were interconnected by suspension in the horizontal (course) and vertical (wale) direction, known as the weft knitting method. The consecutive rows of loops can be reshaped by external or internal forces in any direction..$^{34,35}$ The Fig. 1c (right) shows enlarged images of the knitted surface printed with conductive inks of PEDO:PSS (top) and AgNPs (bottom). We observed that the ink is added from the surface of the fabric and then absorbed from the inside of the fiber bundle to the surface by a capillary phenomenon called "wicking". When conductive ink is printed on top of the fabric, the ink penetrates into the individual fibers and is fixed in the interior of the fiber bundles as the ink dries. Therefore, the small gaps $(<10 \mu \mathrm{m})$ between fibers were filled with the conductor inks, and the conductive paths were formed among the fiber bundles. While the gaps between individual fibers were filled with the inks, the larger spaces between the bundles of fibers were not filled with the ink because the fluid transported, penetrated, and remained only in the fiber bundles. ${ }^{19}$ Thus, the loop structure of the knitted textile was not immobilized, and the textile's softness and deformability were maintained. As shown 
(a)

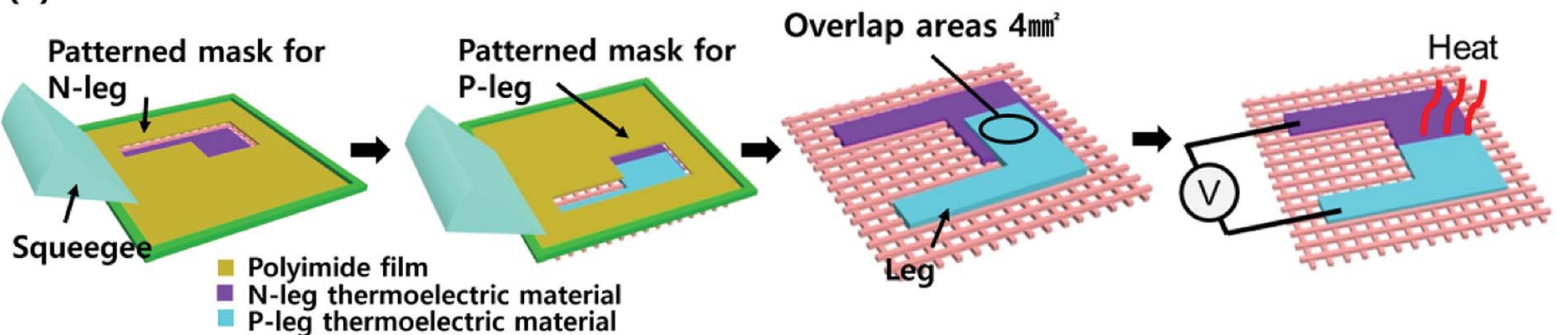

(b)
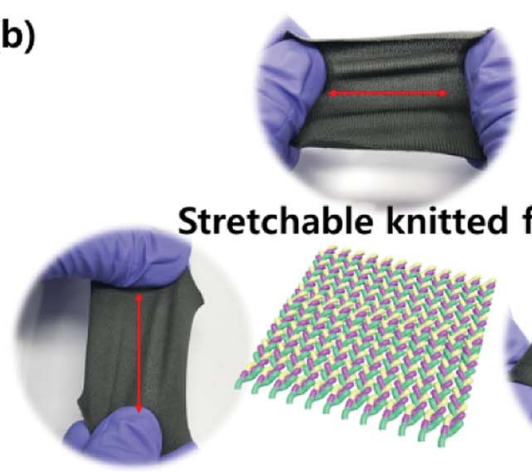

(d)

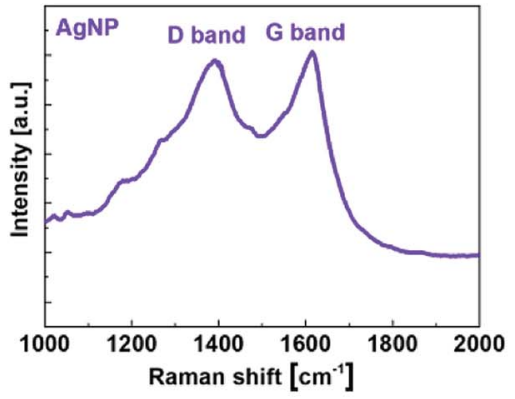

(c)

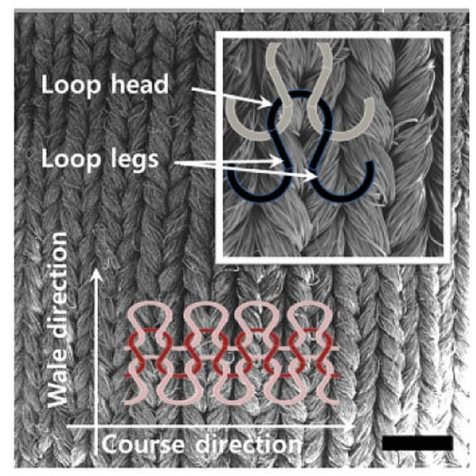

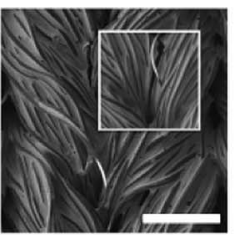

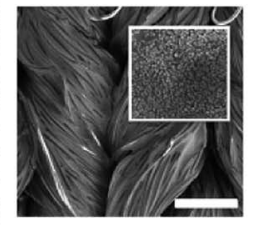

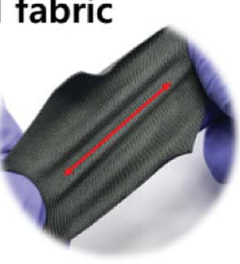

(e)

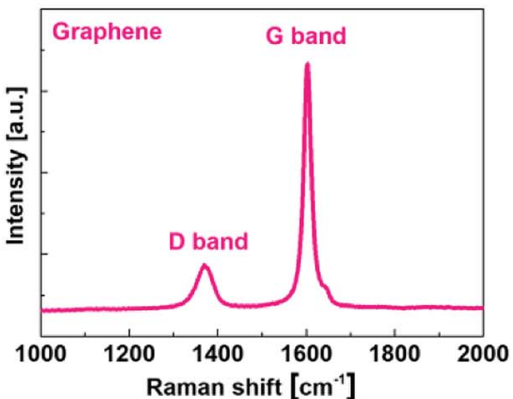

(f)

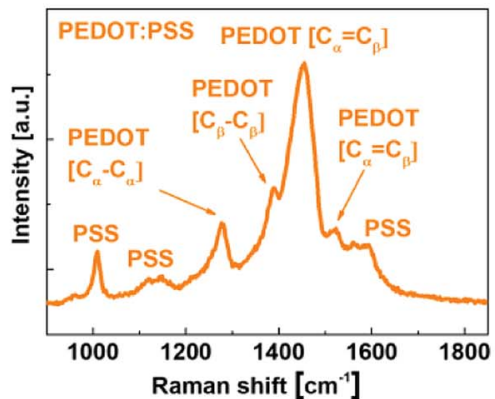

Fig. 1 Schematic illustration of a stencil-printing method, structural characteristics of the stretchable substrate, and material analysis of thermoelectric inks. (a) Fabrication process of the sensor with stencil-printing technique. First, the $\mathrm{N}$-leg (n-type thermoelectric material) was printed onto the textile substrate, and then the P-leg ( $p$-type thermoelectric material) was printed with an overlapped area of $4 \mathrm{~mm}^{2}, \mathrm{using}_{\mathrm{polyimide}}$ patterned masks for both the $\mathrm{N}$-and P-legs. (b) Images of the multi-axis stretchable knitted fabric. (c) SEM images of a pristine knitted fabric illustrated with the knit structure configuration of a loop head and two loop legs (left). Additional SEM images show an enlarged surface with the composite of PEDOT:PSS doped with DMSO (top right) and silver nanoparticles (AgNPs) (bottom right) on knitted fabric. Scale bars are $200 \mu \mathrm{m}$. Raman spectra of AgNPs (d), graphene (e), and PEDOT:PSS doped with DMSO (f).

(a)

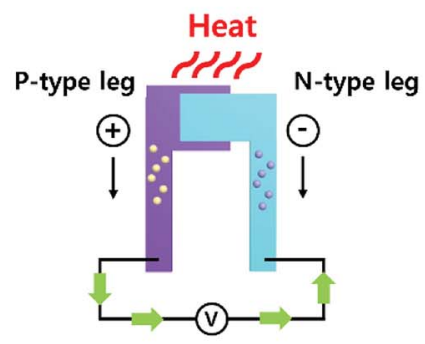

(b)

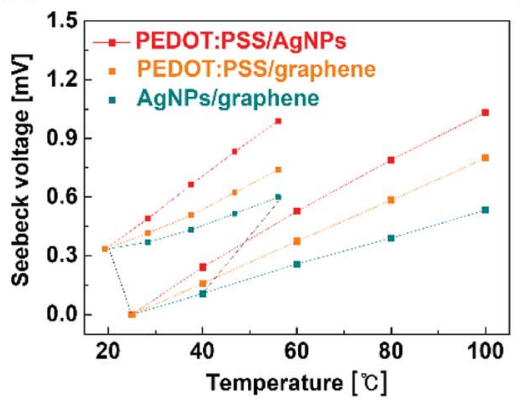

(c)

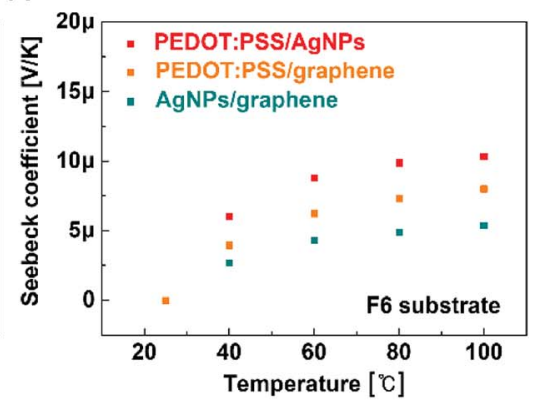

Fig. 2 Thermoelectric properties and output characteristics of the temperature sensor. (a) Principle of the thermoelectric temperature sensor. (b) Output Seebeck voltage and (c) Seebeck coefficient of various thermocouples on the knitted fabric. 
(a)

(b)

(e)

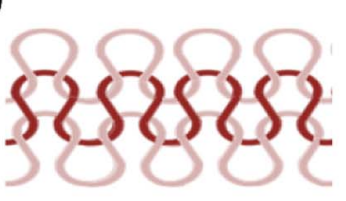

Normal loop

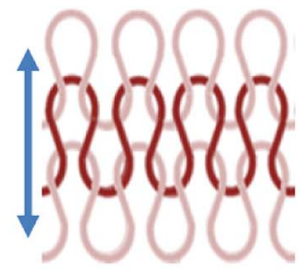

Elongated loop

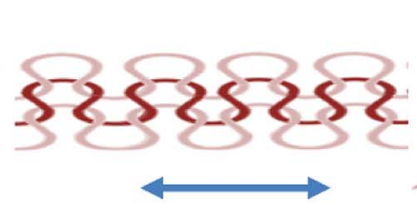

Widened loop

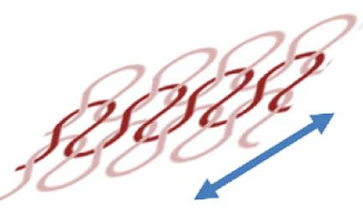

Distorted loop
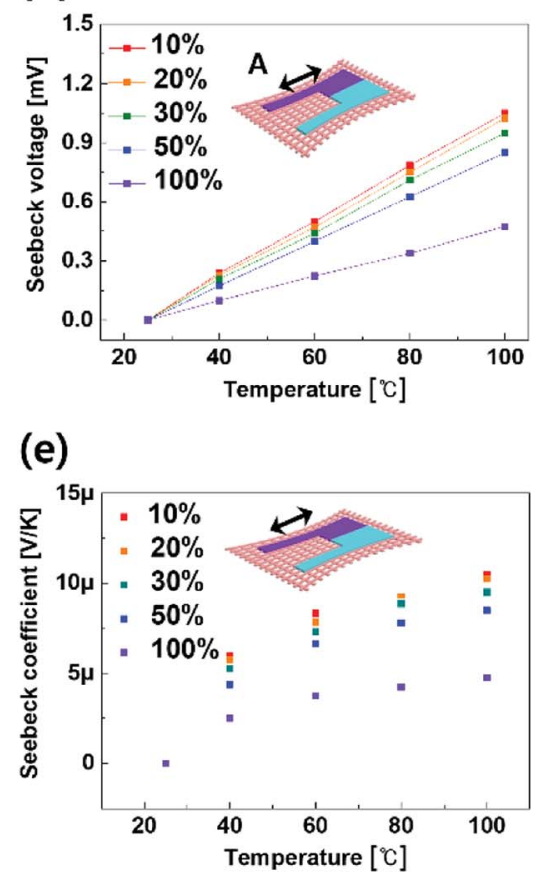

(c)

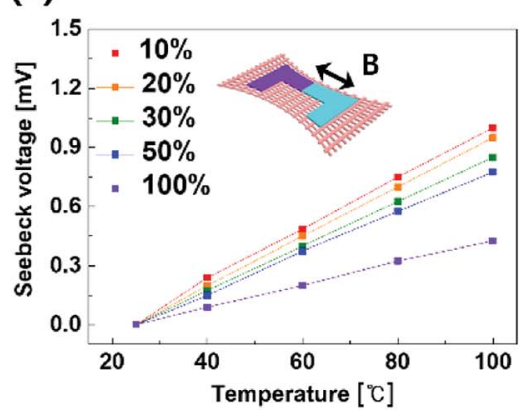

(d)

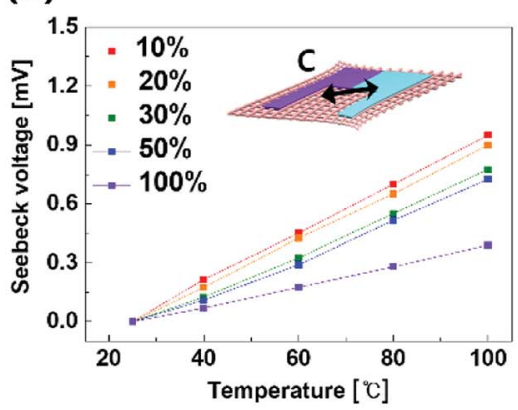

(f)

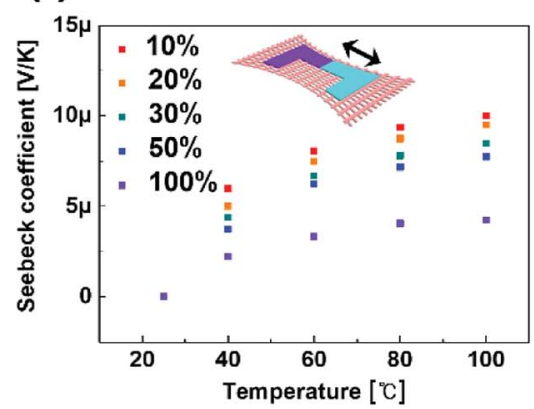

(g)

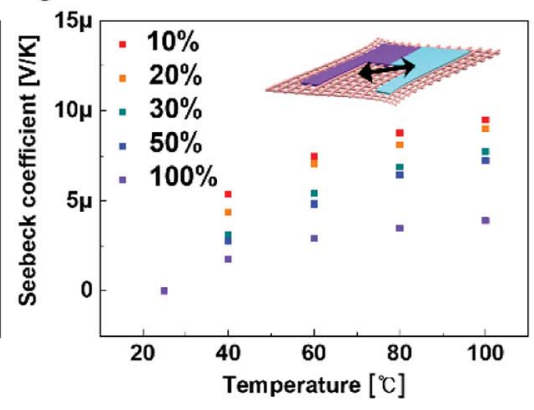

Fig. 3 Schematic diagram of the corresponding deformations of knitted loops and thermoelectric performance of the temperature sensor in relation to stretching deformation. (a) Knitted loops allow elongated, widened, and distorted geometry. Output Seebeck voltage of thermocouple (fabricated with AgNPs and PEDOT:PSS) when (b) elongated, (c) widened, and (d) distorted. Seebeck coefficient of thermocouple (fabricated with AgNPs and PEDOT:PSS) when (e) elongated, (f) widened, and (g) distorted.

in Fig. 1d-f, we measured the Raman spectra of the printed thermoelectric materials using a laser with a wavelength of $532 \mathrm{~nm}$. The Raman spectra of AgNPs with the G and D bands are shown in Fig. 1d. In all the Raman spectra, similarly, two dominant peaks for the $\mathrm{G}$ and $\mathrm{D}$ bands can be observed for graphene ink (Fig. 1e). The D band is due to the breathing mode of the $k$-point phonons of $\mathrm{Ag}_{\mathrm{g}}^{1}$ symmetry, and the $\mathrm{G}$ band corresponds to the first-order scattering of $E_{g}^{2}$ phonons. ${ }^{36}$ Fig. if shows the Raman spectra of conductive polymer PEDOT:PSS, which were reported in a previous article. ${ }^{37}$ The peaks of PEDOT are observed at $1259 \mathrm{~cm}^{-1}$ with the inter-ring stretching vibrational mode, at $1369 \mathrm{~cm}^{-1}$ in stretching mode, and at $1511 \mathrm{~cm}^{-1}$ in asymmetrical mode. ${ }^{30,31}$

Fig. 2a illustrates the principle of thermoelectricity used in the temperature sensor. According to the Seebeck effect, the characteristic of thermoelectric materials is represented by the ratio of the generated thermoelectric voltage, $\Delta V$, to the temperature gradient, $\Delta T$ as $\Delta V=\Delta S T$. Note that $S$ is the Seebeck coefficient, which represents the thermoelectricity of a specific material. Since the sensitivity of the temperature sensor is determined by the thermoelectric conversion efficiency of the materials, a high Seebeck coefficient is necessary for a high-performance temperature sensor. The thermoelectric temperature sensor utilized the thermoelectric voltage generated by the charge exchange from the relative temperature difference at the junction of the semiconductor and/or the metal, which have different electrical characteristics. Therefore, in the fiber-based temperature sensor presented in this study, the junction is the sensing part, which is in contact with the heat source to be measured and with the area where the thermoelectric voltage is generated. As well, we used stencil printing methods that enable large-area, simple, and low-cost processes with solution-based inks of AgNPs, graphene, and PEDOT:PSS. Previous works reported that the work-function values of conductive inks of AgNPs, graphene, and PEDOT:PSS are 4.3, 4.5 and $5 \mathrm{eV}$, respectively. ${ }^{30,31}$ To evaluate the device performance of our thermoelectric materials, heat from two sources, one at room temperature and the other at a higher temperature, was applied to two electrodes to provide a temperature gradient. A plate heat source was used to supply the thermal stimulus, and the generated voltage was read by a high-resolution digital multimeter. We characterized the sensing property of sample 
thermoelectric temperature sensors fabricated with three different combinations of patterned thermoelectric materials. For the first and second combinations, the PEDOT:PSS works as the p-type thermoelectric material, and the AgNPs and graphene as n-type thermoelectric materials. The last device is composed of AgNPs and graphene as the p- and n-type thermoelectric materials, respectively. The measured output voltage and the Seebeck coefficient are shown in Fig. 2b and c, respectively. The thermocouples of PEDOT:PSS and AgNPs, PEDOT:PSS and graphene, and AgNPs and graphene can each achieve output voltage of $1.1 \mathrm{mV}, 0.8 \mathrm{mV}$, and $0.53 \mathrm{mV}$ at temperature differences of $100 \mathrm{~K}$. Among these devices, the PEDOT:PSS and AgNPs thermocouple temperature sensor showed the best performance. We also verified that the printed thermoelectric temperature sensors exhibit linear temperature dependence in a high-temperature range $\left(\sim 100{ }^{\circ} \mathrm{C}\right)$ as well as at low temperatures $\left(30-60{ }^{\circ} \mathrm{C}\right)$ (as shown in the inset).

The stretchability of the devices is an important aspect to consider when designing wearable sensor applications. Knitted structures show excellent elasticity in comparison to other types of textiles due to the number of interdependent loops; the space in each loop allows such fabrics to be elongated, widened, or distorted by external or internal forces, as shown Fig. $3 \mathrm{a} .{ }^{35} \mathrm{We}$ define the stretching behaviour of the knitted substrate as having $A, B$, and $C$ directions. The $A$-direction is parallel with fiber bundles, the $B$-direction is perpendicular to fiber bundles, and $C$-direction is a diagonal of the fiber bundles. To investigate the thermoelectric-strain-sensing properties, the output voltage and Seebeck coefficient were traced under various strain condition. Fig. $3 \mathrm{~b}-\mathrm{g}$ shows the Seebeck voltage and coefficients of the stretchable temperature sensor measured under a range of stretching from $10 \%$ to $100 \%$ in these three directions. Under conditions of low stretching (10-20\%), the output voltage decreases from $1.03 \mathrm{mV}$ to $1.03 \mathrm{mV}, 0.97 \mathrm{mV}$, and $0.94 \mathrm{mV}$ in the $A, B$ and $C$ directions, respectively. However, under conditions of $100 \%$ stretching and high temperature $\left(\sim 100{ }^{\circ} \mathrm{C}\right)$, the output voltage decreases from $1.03 \mathrm{mV}$ to $0.475 \mathrm{mV}, 0.425 \mathrm{mV}$, and $0.39 \mathrm{mV}$ in the $A, B$ and $C$ directions, respectively. That is, the output voltage of the thermoelectric sensor pattern decreased $1 \%$ and $5 \%$ across the overall temperature range under $A$ direction strains of $10 \%$ and $20 \%$, respectively. With increased stretching, the voltage decreased linearly, and with $100 \%$ stretching, it dropped to $50 \%$ of the initial voltage [Fig. S1, ESI $\dagger$. This can be explained by the structural deformation development of the knit loop structure with fiber bundles printed with conductive inks when stress was applied. Initially, the conductive ink on the textile substrate was well integrated with the fibers and stress was relieved by deformation of the textile structure. The deformations continuously undergo slippage between the arc segments and legs of loops. Then, the fiber bundles of the textile have a curved shape that is straightened when the strain is increased. This occurs because the ink selectively fills the small gaps within each fiber bundle and fails to fill the larger gaps between fiber bundles. Eventually, the fiber bundles are stretched resulting in damage to the conductive paths of the printed materials on the fibers due to cracking. Microscopic images of the fabric in its initial state and under $100 \%$ stretching are shown in Fig. S2. $\dagger$ Under $B$-and $C$ direction strain, the output voltage showed 5-fold and 10-fold decreases in comparison with the $A$-direction strain after $10 \%$ stretching. This can be explained by the fact that force applied in the $B$ and $C$ directions is directly delivered to the fiber bundles by straightening and stretching, and it easily breaks the conductive paths among the fiber bundles. These results suggest that there is a limit to the inherent stretchability of conductive inks as thermoelectric materials. Therefore, for further improvement of the stability of the Seebeck effect of such materials under stretching, the adoption of various stretchable conductive composite inks could be considered. ${ }^{3}$ This would allow that the combination of fibers and printed inks to easily accommodate deforming movements, such as bending, straightening, and stretching.

In addition, stability and durability are crucial for practical wearable device applications. We conducted a range of cyclic tests to assess the durability of the thermoelectric temperature sensors, and the results demonstrate their excellent stability. As shown in Fig. 4, the Seebeck voltage and coefficient of the thermoelectric temperature sensor was measured as a function of a range of repeated cycles of $20 \%$ strain. We found that the output Seebeck voltage was stable up to 800 cycles, only decreasing $7 \%$ from the initial output voltage. These results demonstrate that the thermoelectric temperature sensor printed on knitted fabric showed good stretchability and linearity as

(a)

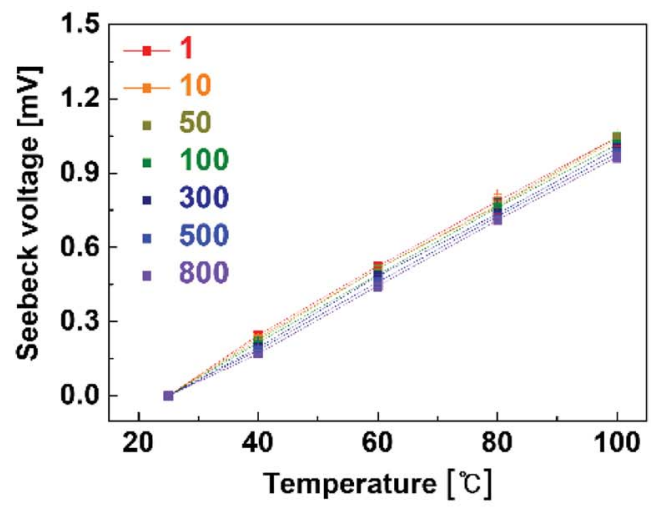

(b)

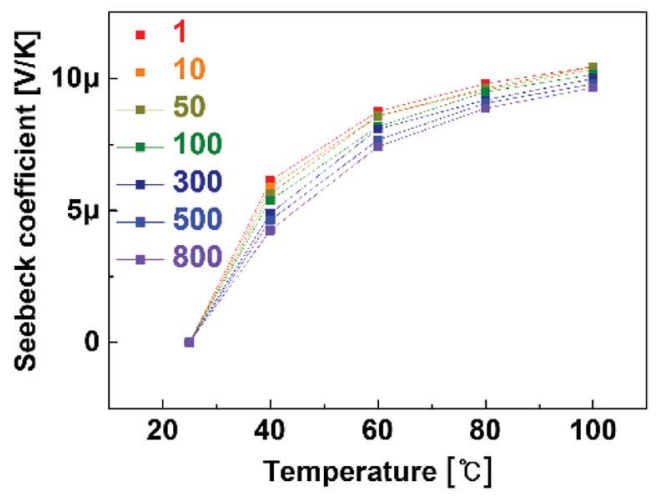

Fig. 4 Temperature sensor characteristics as a function of stretching cycles at 20\%. (a) Output Seebeck voltage (b) Seebeck coefficient. 
(a)

(b)

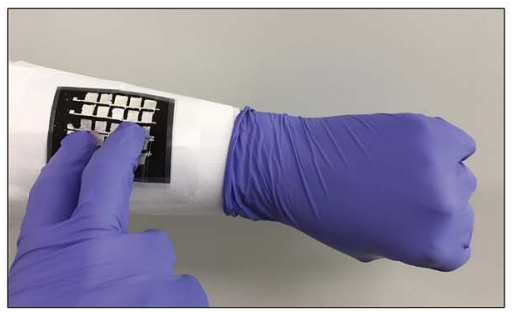

(d)

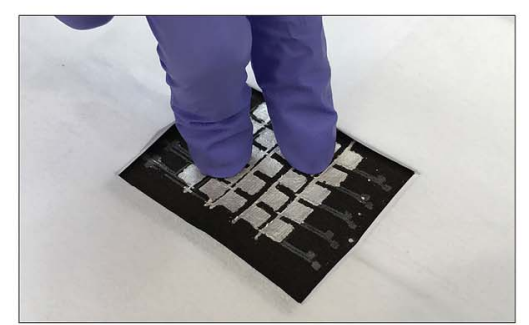

(c)

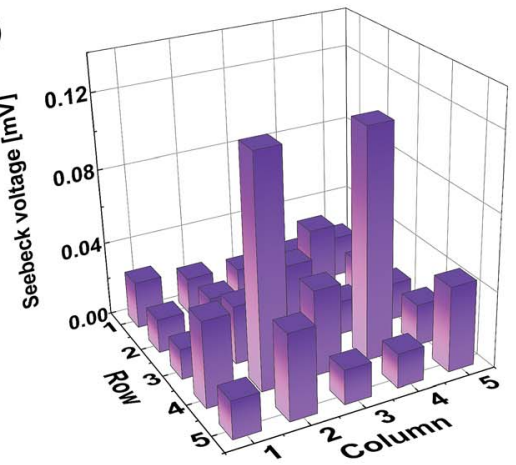

(e)

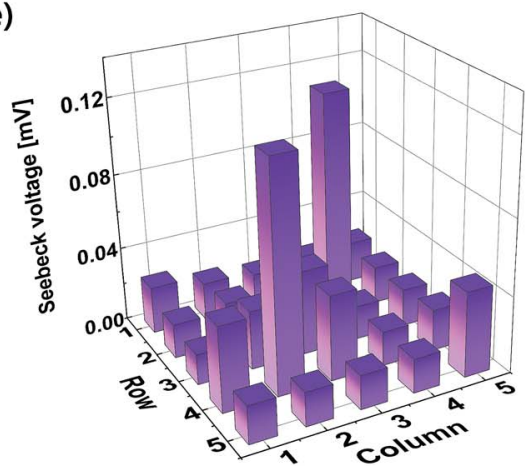

Fig. 5 (a) Schematic layout and ( $b$ and d) optic images of the wearable $5 \times 5$ temperature sensor array, showing that the surface of the array is locally touched by fingers on the specific areas. (c and e) Output Seebeck voltage mapping and corresponding temperature distribution.

well as thermoelectric stability and durability, indicating its advantages for wearable applications. The thermoelectric properties of our temperature sensor do not show remarkable performance in comparison to those using previously reported inorganic materials, such as $\mathrm{Bi}, \mathrm{Te}$, or $\mathrm{Sb}^{38-42}$ However, in the cyclic test, we successfully demonstrated that the stretchable thermoelectric temperature sensor system achieves excellent durability. Moreover, it does not require a high-temperature annealing process to activate the materials, and only requires a low-temperature process $\left(\sim 120{ }^{\circ} \mathrm{C}\right)$ to evaporate the solvent material after printing.

Detecting the position of heat stimuli, which requires a matrix of a temperature sensor, is an important function of wearable devices. To demonstrate a potential application of the stretchable printed temperature sensor, we tested the real-time response of the temperature sensor with 25 sensor arrays. Fig. 5a shows the schematic of the 5 by 5 sensor arrays with the unit areas of $25 \mathrm{~mm}^{2}$, including thermoelectric junctions and transmission lines printed on the stretchable knitted fabric. The sensory system was connected to a LabVIEW-based evaluation system for the spatial imaging profile. When the sensor array was exposed to a gentle finger touch to introduce a local stimulus, it responded to the temperature changes of the single pixel with the real-time mapping arrays, as shown in Fig. $5 \mathrm{c}$ and e. The noise level on the Seebeck voltage was relatively low compared with the local stimulus from the finger due to the sufficient distance between unit cells. For wearable sensors and devices, the low power-driven sensor devices need to be driven by a limited-capacity battery or an energyharvesting device, and these sensors and portable electronic devices must be installed regardless of time and space or attached to the user's body. In addition, the thermoelectric temperature sensor measures the temperature difference with self-generated voltage, so no additional power source is required. Our devices, fabricated on flexible, conformable, and breathable textile substrates by solution-based processes have been demonstrated to have excellent detection capabilities and great potential for application in wearable electronics. In future studies, several strategies for improving the performance will be investigated such as optimizing the doping level of PEDOT:PSS, 
increasing the number of coating processes for the p-type and ntype thermoelectric materials, and optimizing device structure.

\section{Conclusions}

In summary, we reported that a knitted textile-based stretchable self-powered temperature sensor was developed that uses the thermoelectric effect. An intrinsically stretchable knitted fabric was employed for a low-cost and mass-production process, and stencil printing was employed with various conductive inks for simple fabrication. The thermoelectric sensor system is composed of printable conductive inks, such as AgNPs, graphene, and PEDOT:PSS, on the knitted fabric. The thermoelectric-based temperature sensor generated an output thermoelectric voltage of $1.0 \mathrm{mV}$ for a temperature difference of $100 \mathrm{~K}$. It exhibited excellent durability in 800 cyclic tests with only a decrease of $7 \%$ of the initial voltage at $20 \%$ strain. Moreover, it showed a linear relationship between the output voltage and the temperature difference across a wide strain range. Considering the simplicity and low-cost of the process, its suitability for large-scale mass production, and the high stability and durability of the produced sensors, we believe this approach will contribute to realizing the industrial production of conformal wearable electronics.

\section{Conflicts of interest}

There are no conflicts to declare.

\section{Acknowledgements}

This research was supported by the Basic Science Research Program through a National Research Foundation of Korea (NRF) grant funded by the Korean Government (no. 2017R1A2A2A05022574) and the research fund of Hanyang University (HY-2017).

\section{References}

1 S. Seyedin, J. M. Razal, P. C. Innis, A. Jeiranikhameneh, S. Beirne and G. G. Wallace, ACS Appl. Mater. Interfaces, 2015, 7, 21150-21158.

2 T. Yang, W. Wang, H. Zhang, X. Li, J. Shi, Y. He, Q. Zheng, Z. Li and H. Zhu, ACS Nano, 2015, 9, 10867-10875.

3 J. Eom, R. Jaisutti, H. Lee, W. Lee, J.-S. Heo, J.-Y. Lee, S. K. Park and Y.-H. Kim, ACS Appl. Mater. Interfaces, 2017, 9, 10190-10197.

4 A. J. Bandodkar, R. Nunez-Flores, W. Jia and J. Wang, Adv. Mater., 2015, 27, 3060-3065.

5 C. Wang, M. Zhang, K. Xia, X. Gong, H. Wang, Z. Yin, B. Guan and Y. Zhang, ACS Appl. Mater. Interfaces, 2017, 9, 1333113338.

6 K. Kim, M. Jung, B. Kim, J. Kim, K. Shin, O.-S. Kwon and S. Jeon, Nano Energy, 2017, 41, 301.

7 Y. Du, K. Cai, S. Chen, H. Wang, S. Z. Shen, R. Donelson and T. Lin, Sci. Rep., 2015, 5, 6411.
8 L. Zhang, S. Lin, T. Hua, B. Huang, S. Liu and X. Tao, $A d v$. Energy Mater., 2018, 8, 1700524.

9 Y. Yang, Z.-H. Lin, T. Hou, F. Zhang and Z. L. Wang, Nano Res., 2015, 5, 888-895.

10 M. Ito, T. Koizumi, H. Kojima, T. Saito and M. Nakamura, J. Mater. Chem. A, 2017, 5, 12068-12072.

11 Y. Xie, T.-M. Chou, W. Yang, M. He, Y. Zhao, N. Li and Z.-H. Lin, Semicond. Sci. Technol., 2017, 32, 044003.

12 T. Vuorinen, J. Niittynen, T. Kankkunen, T. M. Kraft and M. Mantysalo, Sci. Rep., 2016, 6, 35289.

13 Q. Hua, J. Sun, H. Liu, R. Bao, R. Yu, J. Zhai, C. Pan and Z. L. Wang, Nat. Commun., 2018, 9, 244.

14 N. Saxena, M. Coric, A. Greppmair, J. Wernecke, M. Pfluger, M. Krumrey, M. S. Bandt, E. M. Herzig and P. MullterBuschbaum, Adv. Electron. Mater., 2017, 3, 1700181.

15 S. Jeon, S.-E. Ahn, I. Song, C. J. Kim, U.-I. Chung, E. Lee, I. Yoo, A. Nathan, S. Lee, K. Ghaffarzadeh, J. Robertson and K. Kim, Nat. Mater., 2012, 11, 301.

16 S. Jeon, I. Song, S. Lee, B. Ryu, S. -E. Ahn, E. Lee, Y. Kim, A. Nathan, J. Robertson and U. -I. Chung, Adv. Mater., 2014, 26, 7102.

17 Q. Li, L.-N. Zhang, X.-M. Tao and X. Ding, Adv. Healthcare Mater., 2017, 6, 1601371.

18 S. L. Kim, K. Choi, A. Tazebay and C. Yu, ACS Nano, 2014, 8, 2377-2386.

19 H. Jin, N. Matsuhisa, S. Lee, M. Abbas, T. Yokota and T. Someya, Adv. Mater., 2017, 29, 1605848.

20 K. Jost, D. Stenger, C. R. Perez, J. K. McDonough, K. Lian, Y. Gogotsi and G. Dion, Energy Environ. Sci., 2013, 6, 26982705.

21 L. M. Castano and A. B. Flatau, Smart Mater. Struct., 2014, 23, 053001.

22 J. Ge, L. Sun, F.-R. Zhang, Y. Zhang, L.-A. Shi, H.-Y. Zhao, H.-W. Zhu, H.-L. Jiang and S.-H. Yu, Adv. Mater., 2016, 28, 722-728.

23 M. Park, J. Im, M. Shin, Y. Min, J. Park, H. Cho, S. Park, M.-B. Shim, S. Jeon, D.-Y. Chung, J. Bae, J. Park, U. Jeong and K. Kim, Nat. Nanotechnol., 2012, 7, 803.

24 C. -L. Choong, M. -B. Shim, B. -S. Lee, S. Jeon, D. -S. Ko, T. -H. Kang, J. Bae, S. H. Lee, K. -E. Byun, J. Im, Y. J. Jeong, C. E. Park, J. -J. Park and U. -I. Chung, Adv. Mater., 2014, 26, 3451.

25 N. T. Tien, S. Jeon, D. -I. Kim, T. Q. Trung, M. Jang, B. -U. Hwan, K. -E. Byun, J. Bae, E. Lee, J. B. -H. Tok, Z. Bao, N. -U. Lee and J. -J. Park, Adv. Mater., 2014, 25, 796.

26 S. Chen, S. Liu, P. Wang, H. Liu and L. Liu, J. Mater. Sci., 2018, 53, 2995-3005.

27 J. Wang, P. Xue, X. Tao and T. Yu, Adv. Eng. Mater., 2014, 16, 565-570.

28 Y. Li, Y. Li, M. Su, W. Li, Y. Li, H. Li, X. Qian, X. Zhang, F. Li and Y. Song, Adv. Electron. Mater., 2017, 3, 1700253.

29 L. Allison, S. Hoxie and T. L. Andrew, Chem. Commun., 2017, 53, 7182-7193.

30 M. Jung, K. Kim, B. Kim, H. Cheong, K. Shin, O.-S. Kwon, J.-J. Park and S. Jeon, ACS Appl. Mater. Interfaces, 2017, 9, 26974-26982. 
31 M. Jung, K. Kim, B. Kim, K.-J. Lee, J.-W. Kang and S. Jeon, Nanoscale, 2017, 9, 17212-17219.

32 M. Vosgueritchian, D. J. Lipomi and Z. Bao, Adv. Funct. Mater., 2012, 22, 421-428.

33 S. K. Sinha, Y. Noh, N. Reljin, G. M. Treich, S. HajebMohammadalipour, Y. Guo, K. H. Chon and G. A. Sotzing, ACS Appl. Mater. Interfaces, 2017, 9, 37524.

34 K. Dong, Y.-C. Wang, J. Deng, Y. Dai, S. L. Zhang, H. Zou, B. Gu, B. Sun and Z. L. Wang, ACS Nano, 2017, 11, 94909499.

35 M.-W. Han and S.-H. Ahn, Adv. Mater., 2017, 29, 1606580.

36 Z. T. Tang, L. Zhang, C. Zeng, T. Lin and B. Guo, Soft Matter, 2012, 8, 9214-9220.
37 S. H. Chang, C.-H. Chiang, F.-S. Kao, C.-L. Tien and C.-G. Wu, IEEE Photonics J., 2014, 6, 8400307.

38 Z. Cao, E. Koukharenko, M. J. Tudor, R. N. Torah and S. P. Beeby, Sens. Actuators, A, 2016, 238, 196-206.

39 Z. Lu, H. Zhang, C. Mao and C. M. Li, Appl. Energy, 2016, 164, 57-63.

40 F. Suarez, D. P. Parekh, C. Ladd, D. Vashaee, M. D. Dickey and M. C. Ozturk, Appl. Energy, 2017, 202, 736-745.

41 C. Dun, C. A. Hewitt, H. Huang, J. Xu, D. S. Montgomery, W. Niew, Q. Jiang and D. L. Carroll, ACS Appl. Mater. Interfaces, 2015, 7, 7054-7059.

42 C. Ahou, C. Dun, Q. Wang, K. Wang, Z. Shi, D. L. Carroll, G. Liu and G. Qiao, ACS Appl. Mater. Interfaces, 2015, 7, 21015-22102. 